\title{
A Partial Ordering of Knots Through Diagrammatic Unknotting
}

\author{
Yuanan Diao, Claus Ernst, and Andrzej Stasiak
}

\begin{abstract}
In this paper we define a partial order on the set of all knots and links using a special property derived from their minimal diagrams. A knot or link $\mathcal{K}^{\prime}$ is called a predecessor of a knot or link $\mathcal{K}$ if $C r\left(\mathcal{K}^{\prime}\right)<C r(\mathcal{K})$ and a diagram of $\mathcal{K}^{\prime}$ can be obtained from a minimal diagram $D$ of $\mathcal{K}$ by a single crossing change. In such a case we say that $\mathcal{K}^{\prime}<\mathcal{K}$. We investigate the sets of knots that can be obtained by single crossing changes over all minimal diagrams of a given knot. We show that these sets are specific for different knots and permit partial ordering of all the knots. Some interesting results are presented and many questions are posed.
\end{abstract}

\section{Introduction}

Let $K$ be a tame knot, that is, $K$ is a collection of several piece-wise smooth simple closed curves in $\mathbb{R}^{3}$. Intuitively, if one can continuously deform a tame knot $K_{1}$ to another tame knot $K_{2}$, then $K_{1}$ and $K_{2}$ are considered the same knot in the topological sense. The corresponding continuous deformation is called an ambient isotopy, and $K_{1}, K_{2}$ are said to be ambient isotopic to each other. The set of all (tame) knots that are ambient isotopic to each other is called a knot type. For a fixed knot (type) $\mathcal{K}$, a knot diagram of $\mathcal{K}$ is a projection of a member $K \in \mathcal{K}$ onto a plane. Such a projection $p: K \subset R^{3} \rightarrow D \subset R^{2}$ is regular if the set of points $\left\{x \in D:\left|p^{-1}(x)\right|>1\right\}$ is finite and there is no $x$ in $D$ for which $\left|p^{-1}(x)\right|>2$. In other words, in the diagram no more than two arcs of $D$ cross at any point in the projection and there only finitely many such crossings. The number of crossings not only depends on the knot type $\mathcal{K}$, it also depends on the geometrical shape of the member $K$ representing $\mathcal{K}$ and the projection direction chosen. The minimum number of crossings in all regular projections of all members of $\mathcal{K}$ is called the crossing number of the knot type $\mathcal{K}$ and is denoted by $\mathrm{Cr}(\mathcal{K})$. For any member $K$ of $\mathcal{K}$, we also write $\operatorname{Cr}(K)=\operatorname{Cr}(\mathcal{K})$. Of course, by this definition, if $K_{1}$ and $K_{2}$ are of the same knot type, then we have $\operatorname{Cr}\left(K_{1}\right)=\operatorname{Cr}\left(K_{2}\right)$. Furthermore, it may be the case that for a member $K$ of $\mathcal{K}$, none of the regular projections of $K$ has crossing number $C r(\mathcal{K})$. A diagram $D$ of a knot $K \in \mathcal{K}$ is minimal if the

1991 Mathematics Subject Classification. Primary 57M25.

Key words and phrases. Knots, links, crossing number, unknotting number.

The presented research was supported in part by a Western Kentucky University faculty scholarship award to C. Ernst and by Swiss National Science Foundation Grant 3152-068151 for A. Stasiak. 
number of crossings in the diagram equals $C r(\mathcal{K})$. We will often call $D$ a minimum projection diagram. A knot is alternating if there is a knot projection in which one encounters over-passes and under-passes alternatingly when travelling along the knot projection. The crossing number of an alternating knot $\mathcal{K}$ can be determined by the fact that any reduced alternating diagram of $\mathcal{K}$ is minimal, for example the diagram of the knot $\mathcal{K}_{1}$ in Figure 1 is minimal.

A knot $\mathcal{K}$ is called a composite knot if a member of it can be obtained by cutting open two non-trivial knots $K_{1}$ and $K_{2}$ and reconnecting the strings as shown in Figure 1. We write $K=K_{1} \# K_{2}$ and call $K_{1}$ and $K_{2}$ the connected sum components. Note that a knot can have more than two connected sum components. A knot $\mathcal{K}$ that is not a composite knot is called a prime knot.

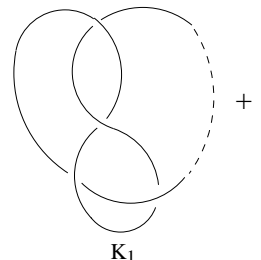

$\mathrm{K}_{1}$

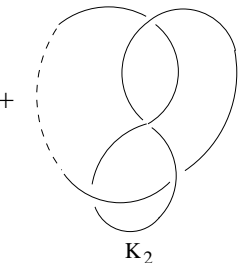

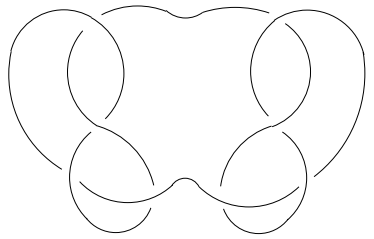

Figure 1. A composite knot $K=K_{1} \# K_{2}$.

Traditionally, knots are tabulated according to their crossing numbers. The crossing number of a knot is a fairly reliable indicator of how complex the knot is. That is, it is usually the case that the larger the crossing number, the more complicated the knot is (in terms of other knot complexity measures). Unfortunately, it is usually difficult to determine the crossing numbers for most large knots. In fact only a few special knot families have known crossing numbers (such as alternating knots, torus knots, and Montesinos knots). A treatment of these knot families can be found in standard textbooks on knot theory such as $[\mathbf{6}, \mathbf{1 6}]$. The crossing numbers of most knots can only be determined if one exhaustively classifies all knot projections with smaller crossing numbers than the given one. This has only been done for knots up to 16 crossings [10] and alternating knots up to 19 crossings [22]. The tables of small knots up to 10 crossings can be found in most knot theory textbooks. See for example [18]. In the case of alternating knots, any two minimum projection diagrams $D$ and $D^{\prime}$ of the same alternating knot $\mathcal{K}$ are flype equivalent, that is, $D$ can be changed to $D^{\prime}$ through a finite sequence of flypes $[\mathbf{1 4}, \mathbf{1 5}]$ (see Figure 2).

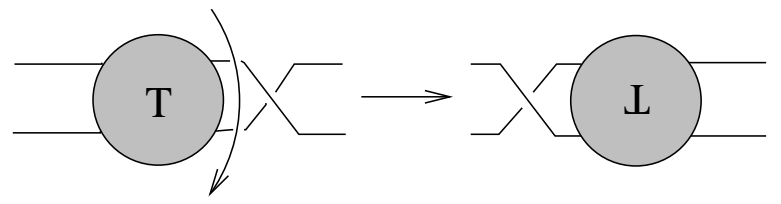

Figure 2. A single flype. $T$ denotes a part of the diagram that is rotated by 180 degrees by the flype.

Let $c$ be a crossing in an alternating diagram $D$. The flyping circuit of $c$ is defined as the unique decomposition of $D$ into crossings $c_{1}, c_{2}, \ldots, c_{m}, m \geq 1$ and 
tangle diagrams $T_{1}, T_{2}, \ldots, T_{r}, r \geq 0$ joined together as shown in Figure 3 such that (i) $c=c_{i}$ for some $i$ and (ii) the $T_{i}$ are minimal with respect to the pattern.

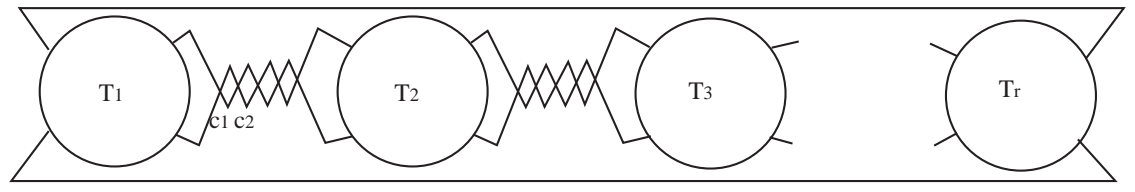

Figure 3. A flyping circuit. Any crossing in the flyping circuit can be flyped to any position between tangles $T_{i}$ and $T_{i+1}$.

Let $D$ be a regular projection diagram of a knot $K$. It is known that $D$ can be made into a diagram of the trivial knot by changing the over/under assignments at some of its crossings. The minimum number of such changes needed is called the unknotting number of the diagram $D$ (and is denoted by $u(D)$ ) and the unknotting number of a knot type $\mathcal{K}$ is the minimum of $u(D)$ where $D$ ranges over all possible regular projections of all tame knot $K$ with type $\mathcal{K}$. The unknotting number of $\mathcal{K}$ (denoted by $u(\mathcal{K})$ ) is a widely studied concept with many known results. See for example $[4,11,17,20,24]$. In this paper, we will introduce and discuss a new concept - called predecessors of a knot - that is related to the unknotting number. On the one hand, instead of considering all possible regular projections of a knot (type) $\mathcal{K}$, we will restrict ourselves to only the minimum regular projections of $\mathcal{K}$. This is similar to the work of Stoimenow [20], where a more restrictive unknotting number is defined by limiting the crossing changes to minimal projections of a knot only. On the other hand, we will consider all the cases when a crossing change is made at each crossing in a minimal projection diagram of $\mathcal{K}$. In other words, predecessors of a given knot include all knots of Gordian distance one from $\mathcal{K}$ via crossing change from a minimum projection diagram of $\mathcal{K}$. In this aspect, the notion is similar but more restrictive than the Gordian distance studied in the literature such as $[\mathbf{2 3}]$.

\section{Predecessors and Siblings of Knots.}

In this paper we investigate the sets of knots that can be obtained by single crossing changes over all minimal diagrams of a given knot. We do this by introducing the concept of predecessors and siblings of a knot. Given a knot (type) $\mathcal{K}$ with a minimum diagram $D$. Recall that in our definition $\mathcal{K}$ may have multiple components. Consider the knot $\mathcal{K}^{\prime}$ obtained from $D$ by a single crossing change. Since the diagram $D^{\prime}$ of $\mathcal{K}^{\prime}$ obtained from $D$ by this single crossing change may or may not be minimal, the minimum crossing number of $\mathcal{K}^{\prime}$ may or may not be strictly less than $\operatorname{Cr}(\mathcal{K})$ (though is is always less than or equal to $C r(\mathcal{K})$ ). We will call $\mathcal{K}^{\prime}$ a predecessor of $\mathcal{K}$ if $C r\left(\mathcal{K}^{\prime}\right)<\operatorname{Cr}(\mathcal{K})$. In case that $\operatorname{Cr}\left(\mathcal{K}^{\prime}\right)=\operatorname{Cr}(\mathcal{K})$, we will say that $\mathcal{K}^{\prime}$ is a sibling of $\mathcal{K}$. For any given knot $\mathcal{K}$, it may have many different minimal diagrams. Consequently, it may have many (distinct) predecessors and siblings obtained from different minimum diagrams of $\mathcal{K}$. Let $\mathcal{K}_{P}$ be the set of all predecessors of $\mathcal{K}$ obtained using all possible minimum diagrams of $\mathcal{K}$, then apparently $\mathcal{K}_{P}$ defines a (rather complicated) topological invariant of $\mathcal{K}$. Similarly, 
the set of all predecessors and siblings of $\mathcal{K}$ (denoted by $\mathcal{K}_{F}$ and called the family of $\mathcal{K}$ ) also defines a topological invariant of $\mathcal{K}$.

Remark 2.1. A predecessor $\mathcal{K}^{\prime}$ of an alternating knot $\mathcal{K}$ may or may not be alternating. However, it can be shown that if $\mathcal{K}$ is alternating and $\mathcal{K}^{\prime}$ is a sibling of $\mathcal{K}$, then $\mathcal{K}^{\prime}$ must be non-alternating. Similarly, a predecessor $\mathcal{K}^{\prime}$ of a prime or non prime knot $\mathcal{K}$ may or may not be prime, see Figure 9.

REMARK 2.2. There are arbitrarily large (in terms of crossing number) knots with only one predecessor. For example the $(n, 2)$ torus knots and any Montesinos knots with Conway symbol $(k, k, \ldots, k)$ have only one predecessor. However it is not clear whether there are knots with only one sibling (or only siblings) and no predecessors. See Question 2.8.

We will now generalize this concept. Assume that there is a sequence of knots $\mathcal{K}=\mathcal{K}_{0}, \mathcal{K}_{1}, \mathcal{K}_{2}, \ldots, \mathcal{K}_{j}$ such that $\mathcal{K}_{i}$ is a predecessor of $\mathcal{K}_{i-1}(i=1, \ldots, j)$, then we will call $\mathcal{K}_{j}$ a $j$-th predecessor of $\mathcal{K}=\mathcal{K}_{0}$. In general, if $j>1$, we may simply call $\mathcal{K}_{j}$ a remote predecessor of $\mathcal{K}$. Furthermore, in case that $\mathcal{K}_{j}$ is the trivial knot, or that $\mathcal{K}_{j}$ has no predecessor, then we will call $\mathcal{K}=\mathcal{K}_{0}, \mathcal{K}_{1}, \mathcal{K}_{2}, \ldots, \mathcal{K}_{j}$ a family trail. We can also define a general family trail by allowing $\mathcal{K}_{i}$ to be a sibling of $\mathcal{K}_{i-1}$ in the above definition.

Let us look at a few examples.

EXAMPLE 2.3. The only predecessor of $3_{1}$ is the trivial knot. The same is true for $4_{1}$. It follows that $\mathcal{K}_{P}$ is not a complete invariant. The only predecessor of $5_{1}$ is $3_{1}$. Hence the family trail of $5_{1}$ is $\left\{3_{1}, 0_{1}\right\}$. Apparently, these three knots have no siblings.

EXAmple 2.4. Let us look at a slightly more complicated case. Shown on top of Figure 4 is the knot 75 . Since there are 7 crossings in a minimum diagram of it, we will have seven possible predecessors or siblings. But some of them are apparently identical by symmetry as shown in the second row of Figure 4 (the first and third). Actually it is easy to see that all predecessors generated by a single row of half-twists are identical. The knot $7_{5}$ has three such row of half-twists, explaining the fact that there are only 3 distinct predecessors. The fact that the predecessors in the figure form the complete predecessor set for $7_{5}$ is explained in the next section (Theorem 3.1). The complete list of family trails of $7_{5}$ is $\left\{7_{5}, 5_{2}, 3_{1}, 0_{1}\right\}$, $\left\{7_{5}, 5_{1}, 3_{1}, 0_{1}\right\},\left\{7_{5}, 5_{2}, 0_{1}\right\},\left\{7_{5}, 3_{1}, 0_{1}\right\}$.

The following theorem is an immediate consequence of the well known fact that any knot projection with $n$ crossings can be made into a projection of the unknot by making at most $n-2$ crossing changes.

TheOREM 2.5. Any given non-trivial knot $\mathcal{K}$ has a general family trail that terminates in the unknot.

We may now define a $\leq$ relation between two knots $\mathcal{K}_{1}$ and $\mathcal{K}_{2}$. We say that $\mathcal{K}_{2}<\mathcal{K}_{1}$ if $\mathcal{K}_{2}$ is in a family trail of $\mathcal{K}_{1}$ and $\mathcal{K}_{2} \neq \mathcal{K}_{1}$. We say that $\mathcal{K}_{2} \leq \mathcal{K}_{1}$ if $\mathcal{K}_{2}$ is in a family trail of $\mathcal{K}_{1}$ and possibly $\mathcal{K}_{2}=\mathcal{K}_{1}$. It is easy to check that this defines a partial ordering for the set of all knots. 


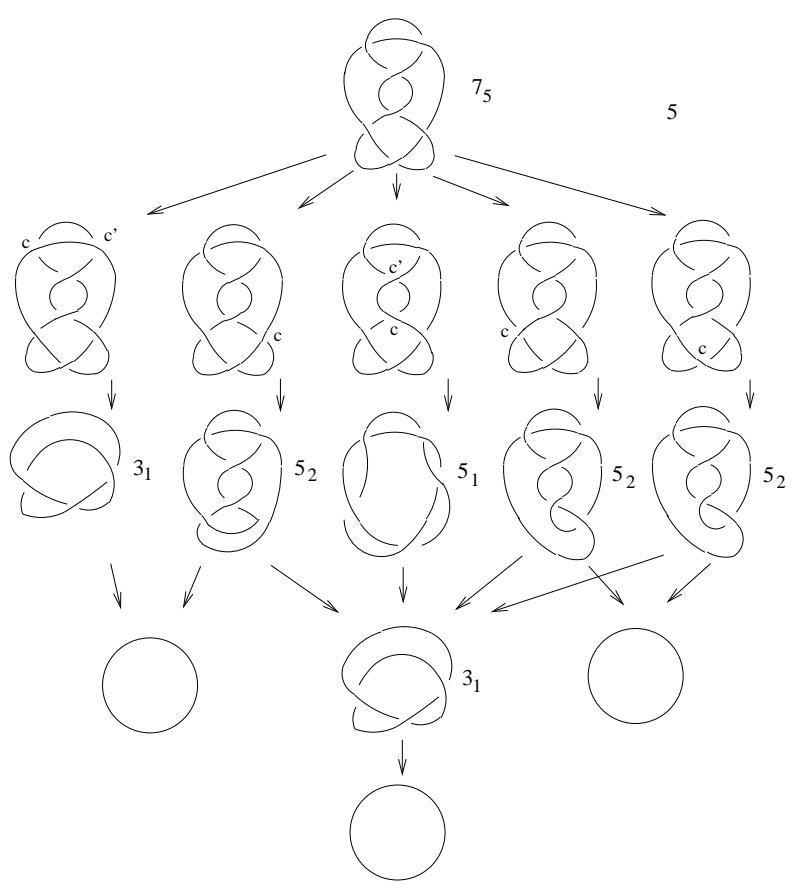

Figure 4. The predecessors of the knot $7_{5}$. The place of the crossing change is marked by $c$. The first and third predecessor in the second row have an identical twin if the crossing change is made at $c^{\prime}$ instead. There are no siblings of $7_{5}$.

Many non-trivial questions arise now at this point. We list a few in the following and will attempt to solve some of them in the next sections.

QUESTION 2.6. Does there exist a non-trivial knot $\mathcal{K}$ with at least one sibling?

A stronger version of the question is the following.

Question 2.7. Does there exist a knot $\mathcal{K}$ that has only siblings?

A negative answer would immediately lead to the conclusion that any nontrivial knot $\mathcal{K}$ has a family trail of length at most $\operatorname{Cr}(\mathcal{K})-1$.

QUeSTION 2.8. Does there exist a knot $\mathcal{K}$ that has exactly one sibling, with or without predecessors?

QUESTION 2.9. Which non-trivial knot $\mathcal{K}$ has no siblings and has the unknot as its only predecessor?

In the case that $\mathcal{K}$ has more than one component, the above question can be rephrased as the following question:

QUESTION 2.10. Which unsplittable link $\mathcal{L}$ has no siblings and has the trivial link as its only predecessor? 
Apparently $3_{1}$ and $4_{1}$ are two knots which have the unknot as the only predecessor. But are there any other such knots? In [21] the concept of an everywhere $n$-trivial knot diagram is discussed. A knot diagram $D$ is everywhere $n$-trivial if changing any $n$ different crossings in $D$ generates a diagram of the unknot. Thus in this language, the standard diagrams of $3_{1}$ and $4_{1}$ are 1-trivial. Furthermore in $[\mathbf{2 1}]$ it is conjectured that the unkot, $3_{1}$ and $4_{1}$ are the only knots possessing 1-trivial diagrams.

QUESTION 2.11. Is there a knot or link with unknotting number one which does not have the unknot as a predecessor?

This question is mentioned in [1] as follows: Does every unknotting number one knot $\mathcal{K}$ have only minimal diagrams in which a single crossing change can unknot $\mathcal{K} ?$

QUESTION 2.12. Which knots have no siblings? In particular, is it true that any alternating knot has no siblings?

Question 2.13. Is it the case that for any given $\mathcal{K}$, a general family trail of it with length $j$ would terminate in the unknot if $j$ is large enough?

As it turns out, there are examples of knots with siblings (examples will be given in the next section). If $\mathcal{K}_{1}$ is a sibling of $\mathcal{K}_{2}$, then of course $\mathcal{K}_{1}$ is also a sibling of $\mathcal{K}_{2}$. So the answer to the above question would be no. However, if we exclude this situation, then the answer to this question is no longer that clear.

QUestion 2.14. How many distinct predecessors and siblings can a knot $\mathcal{K}$ have? What is an upper bound of this number? In particular, is this number bounded above by $\mathrm{Cr}(\mathcal{K})$ ?

We end this section with one more observation.

REMARK 2.15. Let $-\mathcal{K}$ be the mirror image of $\mathcal{K}$. Then the predecessors (siblings) of $-\mathcal{K}$ are the mirror images of the predecessors (siblings) of $\mathcal{K}$. It follows that if $\mathcal{K}$ is achiral then either the predecessors (siblings) are achiral or they will show up as chiral pairs. This can be seen as follows: If $D$ is a minimal diagram of $\mathcal{K}$ then the mirror image $D^{\prime}$ of $D$ is also a minimal diagram of $-\mathcal{K}$. Hence the predecessors (siblings) of $-\mathcal{K}$ are the mirror images of the predecessors (siblings) of $\mathcal{K}$.

\section{Knots with or without Siblings}

In this section, we will first show an example of a non-trivial (multiple components) knot with a sibling (shown in Figure 5). This we can prove directly by using some well known results about alternating knots and linking numbers. We will then show an example for the case of one component knots. We show this by using the knot determination program Knotscape developed by J. Hoste and M. Thistlethwaite [9]. These examples give a positive answer to Question 2.6 and validate Question 2.12 posted in the last section. 


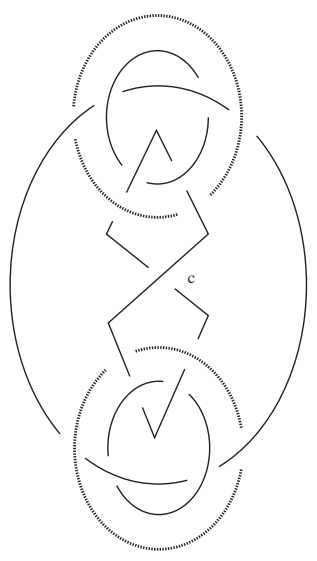

Figure 5. A 17 crossing knot with 6 components: Changing the under/over pass at crossing $c$ results into a sibling.

If one changes the under/over pass at the crossing labelled $c$ in the non-trivial knot $\mathcal{K}$ shown in Figure 5, then the knot $\mathcal{K}^{\prime}$ obtained has the same crossing number as $\mathcal{K}$. This can be seen as follows: The knot has 6 components. A linking number argument between the components shows that there are at least 16 crossings. Suppose $\mathcal{K}$ has a diagram with 16 crossings, then deleting the two dashed thick components must reduce the diagram by at least 8 crossings (because there are at least 8 crossings between the removed components and the remaining components since the total linking number between among them is 4). However, the projection of the remaining components is a reduced alternating diagram with 9 crossings. Thus it must have at least 9 crossings. Therefore, a 16 crossing diagram does not exist. In other word, $C r(\mathcal{K})=17$. Once we make a crossing change at $c$, a similar argument can be applied to $\mathcal{K}^{\prime}$ to shows that $\operatorname{Cr}\left(\mathcal{K}^{\prime}\right)=17$ as well.

To find knots with siblings one can use the knot table compiled by J. Hoste, M. Thistlethwaite and J. Weeks [10]. For an alternating and reduced diagram, it is easy to see the minimum crossing number. However, once a crossing change is made, the diagram becomes non-alternating and it is no longer easy to tell its crossing number and the knot table becomes essential. First one writes down the Dowker-Thistlethwaite code [8] of a knot, and then one makes a crossing change (which means changing the sign of a single entry in the DT code obtained) and looks the changed code up in the knot table using Knotscape [9]. It turns out that small knots mostly have no siblings. Evidence collected in $[2]$ suggests that all knots with less or equal to 9 crossings have no siblings. However we find that, as the crossing number increases, knots with siblings become more common. Such examples involve mostly non-alternating diagrams. Below we give two examples. On the left side of Figure 6 is the Perko knot $\left(10_{161}\right.$ in the Rolfsen table [18] or $10 n 31$ in the Knotscape table) and on the right side of Figure 6 is a sibling of the Perko knot $\left(10_{145}\right.$ in the Rolfsen table and $10 n 14$ in the Knotscape table). In fact, the other knot diagram of the Perko knot would leads to a different sibling of it, namely the knot $10_{139}$ in the Rolfsen table (10n27 in the Knotscape table). We will leave this to our reader to verify. On the left side of Figure 7 is a 16 crossing alternating knot (knot number 16a267637 in the Knotscape table whose DT code is 
(6 820216242228263243018101412 )), and on the right side of Figure 7 is a sibling of it by a single crossing change (knot number $16 n 172116$ in the Knotscape table whose DT code is (6 82021624222826 - 32430181014 12)).
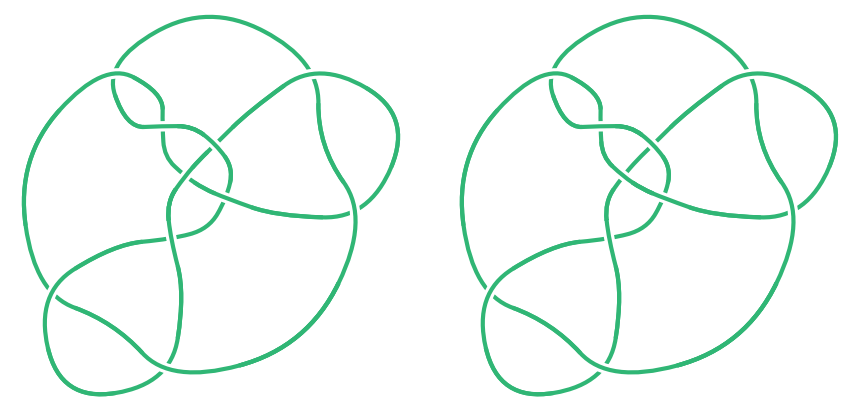

Figure 6. A sibling of the Perko knot: on the left is a diagram of the Perko knot and on the right is the knot $10_{145}$ (knot number $10 n 14$ in the Knotscape table).
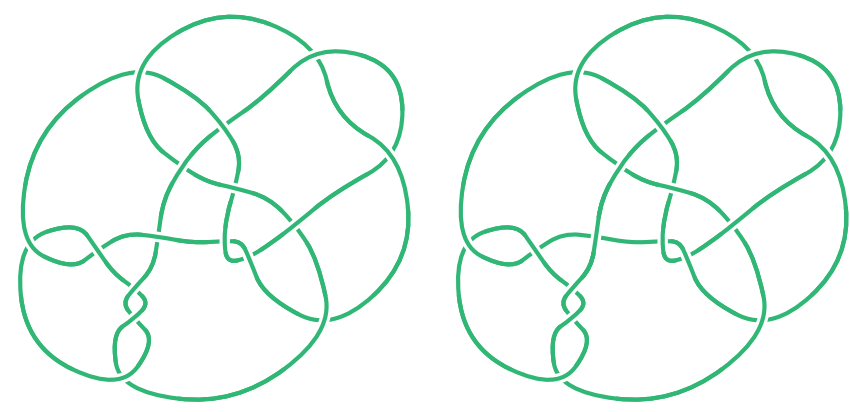

FiguRe 7. A one component 16 crossing alternating knot with a non-alternating sibling. The crossing leading to this sibling is visible to the reader.

We will now state and prove a few theorems. The following theorem answers Question 2.14 affirmatively in the case of alternating knots.

TheOREM 3.1. If $\mathcal{K}$ is an alternating knot then the family $\mathcal{K}_{F}$ of $\mathcal{K}$ (the set of all predecessors and siblings of $\mathcal{K}$ ) can be computed from any minimal diagram of $\mathcal{K}$. Moreover the cardinality of $\mathcal{K}_{F}$ is bounded above by the number of flyping circuits in a minimal diagram of $\mathcal{K}$. In particular, the cardinality of $\mathcal{K}_{F}$ is bounded above by $\mathrm{Cr}(\mathcal{K})$.

Proof. Any minimal diagram $D$ of an alternating knot or link $\mathcal{K}$ can be changed to any other minimal diagram $D^{\prime}$ of $\mathcal{K}$ by a finite sequence of flypes $[14, \mathbf{1 5}]$. To show that $\mathcal{K}_{F}$ can be computed from a single minimal diagram of $\mathcal{K}$ it suffices to show that if $D^{\prime}$ is obtained from $D$ by a single flype then the set of 
direct predecessors obtained from $D^{\prime}$ is the same as the set of direct predecessors obtained form $D$. Let $L$ be the knot obtained from $D$ by changing a single crossing $a$. It is easy to see that $L$ can also be obtained from $D^{\prime}$ by changing the same crossing $a$ in $D^{\prime}$. (Note, if $a$ is the crossing in $D$ eliminated by the flype in $D$ then we will simply treat the crossing created by the flype in $D^{\prime}$ as $a$.) The second part of the theorem follows from the observation that changing any one crossing $c_{i}$ in the same flyping circuit results in flype equivalent predecessors or siblings (which are identical knots).

REMARK 3.2. If a crossing $c$ in an alternating minimal diagram $D$ of $\mathcal{K}$ has a flyping circuit of $c$ with more than two crossings then changing any crossing in the flyping circuit results into a strict predecessor with exactly 2 fewer crossings.

TheOREM 3.3. If $\mathcal{K}$ is a rational (two bridge) knot then $\mathcal{K}$ has no siblings and all predecessors of $\mathcal{K}$ are also rational. Moreover if $\mathcal{K}^{\prime}$ is a predecessor of $\mathcal{K}$ then $\operatorname{Cr}\left(\mathcal{K}^{\prime}\right) \leq \operatorname{Cr}(\mathcal{K})-2$. If $\mathcal{K}$ has a continued fraction expansion of length $k$ then $\mathcal{K}$ has at most $k$ predecessors. In particular the number of predecessors of $\mathcal{K}$ is strictly less than $\mathrm{Cr}(\mathcal{K})$ and any $j$-th predecessor of $\mathcal{K}$ is the unknot if $j \geq C r(\mathcal{K}) / 2$.

Proof. Let $\mathcal{K}$ have the continued fraction expansion $\left\langle c_{1}, c_{2}, \ldots, c_{k}\right\rangle$. Changing any crossing in the 4-plat given by the continued fraction gives a diagram that is still a 4-plat. Thus any predecessor of $\mathcal{K}$ is also rational. Changing any crossing within the $c_{i}$ crossings given by $c_{i}$ results in the same predecessor and thus the number of predecessors of $\mathcal{K}$ is at most $k$. If $c_{i}>2$ for all $i$ (or if $c_{i}=2$ and $1<i<k$ ) then it is obvious that the number of crossings of the predecessor is reduced by exactly 2 . In most other cases the number of crossings reduces by more than 2. Say we change a crossing within the $c_{i}$ crossings given by $c_{i}$ then we might as well change one of the outermost crossings in $c_{i}$. Figure 8 shows that the number of crossings goes done by at least two. Finally if there is a $c_{i} \geq 2$ then the number of predecessors of $\mathcal{K}$ is strictly less than $\operatorname{Cr}(\mathcal{K})$. If all $c_{i}=1$ then changing the crossing in $c_{1}$ produces the same predecessor as changing the crossing in $c_{2}$. Again the number of predecessors of $\mathcal{K}$ is strictly less than $\operatorname{Cr}(\mathcal{K})$. The last statement of the theorem is obvious.
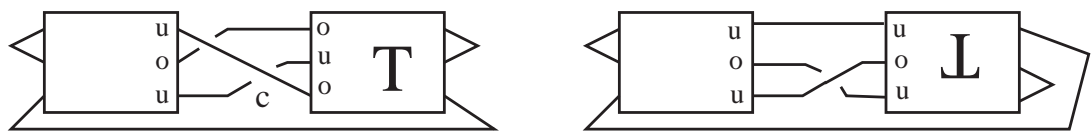

Figure 8. The crossing $c$ is changed in the diagram on the left to make it non-alternating. The part of the diagram labelled $T$ is flipped over. This eliminates one crossing. The labels $u$ and $o$ indicated that the first crossing encountered when following the curve of the knot into the box is encountered by an over or underpass. Thus the diagram on the right is still non-alternating and reduces further. Thus the crossing number decreases by at least two.

REMARK 3.4. In $[\mathbf{7}, \mathbf{2 3}]$ a criteria is given for 4-plats that can be obtained from each other by a single crossing change (or strand passage). However this criteria includes crossing changes in non minimal diagrams. In fact from any 4-plat 
one can obtain infinitely many others by a strand passage. If one just looks at the predecessors of a 4-plat then these can be obtained from re-expanding the continued fractions of the form $\left\langle c_{1}, \ldots, c_{i}-2, \ldots, c_{k}>\right.$ into standard form, where $1 \leq i \leq k$.

TheOREM 3.5. If $\mathcal{K}$ is an alternating Montesinos knot, then $\mathcal{K}$ has no siblings. Furthermore, any predecessor of $\mathcal{K}$ is also a Montesinos knot or it is a connected sum of 2-bridge knots.

Proof. An alternating Montesinos knot $\mathcal{K}$ has a minimal diagram that exhibits the rational tangle structure of $\mathcal{K}$. Changing a crossing in one of these rational tangles, say the tangle $T$, leads to a diagram of the predecessor $L$ that is identical to the diagram of $\mathcal{K}$ outside of the tangle $T$ and has a simpler rational tangle $T^{\prime}$ instead of $T$ with fewer crossings. If $T^{\prime}$ is not the infinity tangle than $L$ is a Montesinos knot. If $T^{\prime}$ is the infinity tangle then $L$ is a connected sum of 2-bridge knots, where each summand is given by one of the rational tangles. Assume that $\mathcal{K}$ has $c$ horizontal twists. If $L$ is obtained by changing one of the $c$ crossings in the row of horizontal twists then if $c>1$ just two of the $c$ crossings are deleted in $L$. If $c=1$ then the changed crossing is absorbed into one of the rational tangles. In this case we obtain a non-alternating knot $L$ whose crossing number is exactly one less than $\operatorname{Cr}(\mathcal{K})[\mathbf{1 3}]$.

REMARK 3.6. The condition that $\mathcal{K}$ is alternating in Theorem 3.5 is necessary. If $\mathcal{K}$ is a non-alternating Montesinos knot then a minimal diagram may not show the rational tangle structure. Moreover there are no readily available methods to draw all minimal diagrams. This makes it very difficult to compute $\mathcal{K}_{F}$. Figure 9 illustrates this. The figure shows three minimal projections of the knot $8_{21}$. In the projection shown at the right side, the structure of the Montesinos knot $((21,21,2-)$ in Conway notation) is clearly visible and not all predecessors of this diagram are prime knots. The projection on the left is the standard diagram in the Rolfsen table [18], here the Montesinos structure is no longer visible. The diagram in the center shows yet another projection. In this projection one of the predecessors obtained is the knot $5_{1}$, which is not a predecessor obtained from any of the other two diagrams. It is easy to find examples of Montesinos knots for which there is a predecessor whose crossing number is exactly one less than the original knot. For example the alternating 9 crossing knot $9_{16}$ ( or $(3,3,3 / 2)$ as a Montesinos knot) has the non-alternating 8 crossing knot $8_{19}$ (or $(3,3,-2)$ as a Montesinos knot) as a predecessor.

The following theorem answers Question 2.10 from the last section completely.

TheOREM 3.7. Let $\mathcal{L}$ be an unsplittable link with $k \geq 2$ components. If $\mathcal{L}$ has no siblings and the only predecessor of $\mathcal{L}$ is the trivial link (of $k$ components), then $k=2$ and $\mathcal{L}$ is the Hopf link.

Proof. First let us consider the case that $k \geq 3$ and let $L$ be a realization of $\mathcal{L}$ with a minimal projection diagram $D$. Let $C_{1}$ and $C_{2}$ be two components of $L$ that have crossings between them. Since $L$ is unsplittable, either $C_{1}$ or $C_{2}$ has intersections with other components in $D$. Say a component $C_{3}$ also has at least one (hence two) crossings with $C_{1}$ in the projection diagram $D$. If the linking number between $C_{1}$ and $C_{2}$ is not zero, then changing a crossing between $C_{1}$ and $C_{3}$ will not 

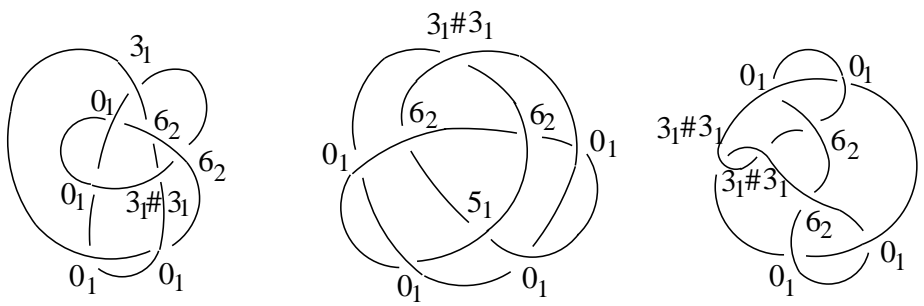

Figure 9. Three different minimal projections of the knot $8_{21}$ : The predecessor obtained at a given crossing is marked at that crossing in the diagrams. The left one is the standard diagram in the knot table. Only the middle diagram yields a 5 crossing predecessor. The diagram on the right is the only (among the three shown here) one in which the three tangles forming the Montesinos knot are visible.

affect that fact, hence the direct predecessor obtained after that crossing change will still have a non-trivial two-component link. If the linking number between $C_{1}$ and $C_{2}$ is zero, then changing a crossing between $C_{1}$ and $C_{2}$ will result in a non-zero linking number ( 1 or -1 ) between $C_{1}$ and $C_{2}$, forming a two-component non-trivial link. So, in either case, there is at least one direct predecessor that is not the trivial link. Thus we must have $k=2$.

Now, again let $C_{1}$ and $C_{2}$ be the two components of $L$. Consider the linking number between $C_{1}$ and $C_{2}$. If this linking number is zero, then changing a crossing between $C_{1}$ and $C_{2}$ will again result in a non-zero linking number, forcing $C_{1}$ and $C_{2}$ into a two-component non-trivial link. On the other hand, if the linking number between $C_{1}$ and $C_{2}$ has absolute value at least 2, then changing a single crossing will still result in a non-zero linking number. Thus the only case left to consider is when the linking number between $C_{1}$ and $C_{2}$ is either one or negative one. If there are only two crossings in $D$, then of course $\mathcal{L}$ is the Hopf link and we are done. If $D$ contains more than two crossings, then either there are $2 m$ crossings between $C_{1}$ and $C_{2}$ with $m \geq 2$, or there are crossings involving only $C_{1}$ or $C_{2}$. In the later case, changing a crossing that does not involve $C_{1}$ and $C_{2}$ will not change the crossing number between $C_{1}$ and $C_{2}$, yielding a non-trivial link as a direct predecessor. In the former case, say the linking number between $C_{1}$ and $C_{2}$ is 1 , then there exactly $m+1$ positive crossings and $m-1 \geq 1$ negative crossings between $C_{1}$ and $C_{2}$. Apparently, a crossing change at a negative crossing will yield a non-trivial link with linking number 2 . The argument is similar if the linking number between $C_{1}$ and $C_{2}$ is -1 . This finishes the proof.

\section{Diagrammatic Unknotting Number}

In this section, we will discuss a concept that can be thought of as a generalization of the unknotting number restricted to minimal diagrams of a knot introduced and studied in $[\mathbf{3}]$ and $[\mathbf{2 0}]$. 
Definition 4.1. For a knot $\mathcal{K}$, a diagrammatic unknotting sequence is a family trail of $\mathcal{K}: \mathcal{K}_{0}=\mathcal{K}, \mathcal{K}_{1}, \mathcal{K}_{2}, \ldots, \mathcal{K}_{n}$ (such that $\mathcal{K}_{i}$ is either a predecessor of $\mathcal{K}_{i-1}$ or a sibling of $\mathcal{K}_{i-1}$ ) that terminates in the trivial knot. We call $n$ the length of such a diagrammatic unknotting sequence. The shortest length over all diagrammatic unknotting sequences of $\mathcal{K}$ is then defined as the diagrammatic unknotting number of $\mathcal{K}$. We will denote it by $u_{D}(\mathcal{K})$.

The concept of $u_{D}$ was first discussed in [3], though worded differently. Recall that a knot $\mathcal{K}$ has unknotting number $u(\mathcal{K})=n$ if there exists a projection of the knot such that changing $n$ crossings in the projection turns the knot projection into the unknot and no projection of $\mathcal{K}$ exists such that changing fewer than $n$ crossings would do this. Equivalently, a knot $\mathcal{K}$ has unknotting number $n$ if $n$ is the least number of crossing changes necessary to change a knot into the unknot, where we can perform the first crossing change in one projection of the knot, then do an ambient isotopy of the resulting projection to a new projection and change a second crossing in that projection. We can do another ambient isotopy before changing a third crossing and so on, see [1]. It follows immediately that for any knot $\mathcal{K}$ we have $u(\mathcal{K}) \leq u_{D}(\mathcal{K})$. It is conjectured that $u(\mathcal{K})=u_{D}(\mathcal{K})$ in $[\mathbf{3}]$.

Now, let $u_{\min }(\mathcal{K})$ be the least unknotting number among all minimal projections of $\mathcal{K}$ as defined in $[\mathbf{2 0}]$, then it is clear that $u(\mathcal{K}) \leq u_{\min }(\mathcal{K})$. One wonders if there is a clear relation between $u_{D}(\mathcal{K})$ and $u_{\min }(\mathcal{K})$. It is obvious that $u_{\min }(\mathcal{K})=1$ if and only if $u_{D}(\mathcal{K})=1$. In the case of two bridge knots, we have the following theorem. But in general, this is a hard question.

TheOREM 4.2. If $\mathcal{K}$ is a 2-bridge knot with unknotting number $u(\mathcal{K})=1$, then $u_{D}(\mathcal{K})=u_{\min }(\mathcal{K})=1$ as well.

This is already established in [21] however the proof below is much shorter.

Proof. In [11] a criteria for 2-bridge knots with unknotting number one is given. In particular $\mathcal{K}$ is a two bridge knot with $u(\mathcal{K})=1$ if and only if $\mathcal{K}$ has a 4plat symbol $\left\langle a, a_{1}, a_{2}, \ldots, a_{r-1}, a_{r}, \pm 2,-a_{r},-a_{r-1}, \ldots,-a_{2},-a_{1}\right\rangle$, where $a$ and the $a_{i}$ 's are integers. It is easy to see that this is equivalent to the alternating diagram $\left\langle a, a_{1}, a_{2}, \ldots, a_{r-1}, a_{r}, 1,1,\left(a_{r}-1\right), a_{r-1}, \ldots, a_{2}, a_{1}\right\rangle$ in the case of 2 and equivalent to the alternating diagram $\left\langle a, a_{1}, a_{2}, \ldots, a_{r-1},\left(a_{r}-1\right), 1,1, a_{r}, a_{r-1}, \ldots, a_{2}, a_{1}\right\rangle$ in the case of -2 . $\left\langle a, a_{1}, a_{2}, \ldots, a_{r-1}, a_{r},-1,1, a_{r}-1, a_{r-1}, \ldots, a_{2}, a_{1}\right\rangle$ is the unknot in the first case and $\left\langle a, a_{1}, a_{2}, \ldots, a_{r-1}, a_{r}-1,1,-1, a_{r}, a_{r-1}, \ldots, a_{2}, a_{1}\right\rangle$ is the unknot in the second case. Thus for any 2-bridge knot with unknotting number one there is an alternating diagram with the unknot as a predecessor.

Since the equality $u_{D}(\mathcal{K})=u(\mathcal{K})=1$ is an if and only if relation for two bridge knots we have the following corollary.

COROllary 4.3. If $\mathcal{K}$ is a 2 -bridge knot with diagrammatic unknotting number $u_{D}(\mathcal{K})=2$, then $u(\mathcal{K})=2$ as well.

In [4], it is shown that the 4 -plat $\mathcal{K}=(5,1,4)$ has the property $u(\mathcal{K})=2$ but it cannot be obtained from any minimum diagram of it. It can be easily shown that $u_{\min }(\mathcal{K})=3$ and $u_{D}(\mathcal{K})=2$. Thus $u_{D} \neq u_{\min }$ in general. The following 
generalization of this example is found in $[\mathbf{2 0}]$. Here we take the arborescent prime knot $\mathcal{K}_{n}$ described by the following symbol in Conway notation:

$$
(((5,1,4),(5,1,4), \ldots,(5,1,4)), 3,3,),
$$

where the rational tangle $(5,1,4)$ is repeated $n$ times, see Figure 10. It is straight forward to see that $u_{D}\left(\mathcal{K}_{n}\right) \leq 2 n+3$. In [20] it is shown that the quantity $u_{\min }\left(\mathcal{K}_{n}\right)-2 n$ cannot be bounded by a constant independent of $n$. Thus we have shown examples of knots where $u_{\min }-u_{D}$ is as large as we desire.
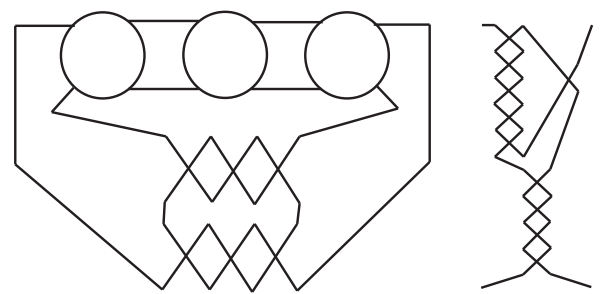

Figure 10. Shown is a diagram of on such knot given by the symbol $(((5,1,4),(5,1,4),(5,1,4)), 3,3$,$) . In the diagram on the$ left there are three disks each of which contains one of the tangles $(5,1,4)$. On the right one such tangle is shown. Since the knot is alternating we did not indicate over- and under passes at the crossings.

We end this section with the following theorem which concerns the cardinality of the set of all knots that belong to some family trails of a given knot.

THEOREM 4.4. There are knots $\mathcal{K}$ with $n$ crossings such that the cardinality of the set of all knots occur in some family trails of $\mathcal{K}$ grows exponentially with respect to $n$.

Proof. Let $\mathcal{K}$ be a 4 -plat with vector symbol $(k, k, \cdots, k)$, where $k$ is an odd positive integer. If $C r(\mathcal{K})=n$ and there are $r k$ 's in the vector $(k, k, \cdots, k)$, then $r k=n$. We can estimate the number of predecessors of $\mathcal{K}$ that also have a 4-plat vector of length $r$ in the following way. Any 4-plat with a vector $\left(k_{1}, k_{2}, \cdots, k_{r}\right)$ where $k_{i}$ is a positive integer of the form $k-2 s$ for some integer $s>0$ is a predecessor of $\mathcal{K}$. Each $k_{i}$ can take on $(k-1) / 2$ different nonzero values giving rise to $((k-1) / 2)^{r}$ different vectors. Since for a 4 -plat, the reversal of the vector does not change the knot type, this results in $1 / 2((k-1) / 2)^{r}$ different predecessors. If we choose $k=5$ and $r=n / 5$ then $\mathcal{K}$ has at least $1 / 2 \times 2^{\sqrt{n} / 5} \approx 1 / 2 \times 1.487^{n}$ different predecessors.

\section{Ending Remarks}

In this paper, we have introduced the concept of predecessors and siblings of a knot and the concept of diagrammatic unknotting number of a knot. We raised many questions along the way. Although we were able to answer a few of these questions, most questions are hard and remain open. We end this paper with a few more remarks. 
Remark 5.1. Computing the family of a non-alternating knot in general is a hard problem since it is hard to find all minimal diagrams of such a knot.

REMARK 5.2. It would be interesting to see if there are knots for which the cardinality of the set of predecessors equals or exceeds the crossing number of the knot.

REMARK 5.3. One may attempt to prove the conjecture $u_{D}=u$ for the rational knots first. Notice that this is true for the simple case of the $(n, 2)$ torus knot, since in this case we already know that $u_{D}=(n-1) / 2$ and $u=(n-1) / 2$. (In general, it is known that the unknotting number for a $(p, q)$ torus knot is $(p-1)(q-1) / 2$ $[12,19]$.)

\section{References}

[1] C. C. Adams, The knot book: An elementary introduction to the mathematical theory of knots,

W. H. Freeman and Co., 1994.

[2] A. Flammini and A. Stasiak, Natural classification of knots, preprint.

[3] J. A. Bernhard, Unknotting numbers and minimal knot diagrams, J. Knot Theory Ramifications 3(1) (1994), pp. 1-5.

[4] S. A. Bleiler, A note on unknotting number, Math. Proc. Camb. Phil. Soc. 96 (1984), pp. 469-471.

[5] G. Burde, Über das Geschlecht und die Faserbarkeit von Montesinos-Knoten, Abh. Math. Sem. Univ. Hamburg 54 (1984), pp. 199-226.

[6] G. Burde and H. Zieschang, Knots, de Gruyter, Berlin, 1986.

[7] I. Darcy, and D. MW. Sumners, A strand passage metric for topoisomerase action, Knots '96: Proceedings of the Fifth MSJ International Research Institute of Mathematical Society of Japan, July 1996, World Scientific, ed. S. Suzuki (1997), pp. $267-278$.

[8] C. H. Dowker and M. Thistlethwaite, Classification of knot projections, Topol. Appl. 16 (1983), pp. 19-31.

[9] J. Hoste and M. Thistlethwaite, Knotscape, http://www. math .utk.edu/ morwen/knotscape.html.

[10] J. Hoste, M. Thistlethwaite, and J. Weeks, The first 1,701,936 knots, Mathematical Intelligencer 20(Fall) (1998), pp. 33-48.

[11] T. Kanenobu and H. Murakami, Two bridge knots with unknotting number one, Proc. AMS., 98(3), 499-502 (1986).

[12] P. B. Kronheimer and T. S. Mrowka, Gauge theory for embedded surfaces, I, Topology 32(4) (1993), pp. $773-826$.

[13] W. B. R. Lickorish and M. B. Thistlethwaite, Some links with nontrivial polynomials and their crossing-numbers, Comment. Math. Helv. 63(4) (1988), pp. 527-539.

[14] W. Menasco and M. Thistlethwaite, The Tait flyping conjecture, Bull. Amer. Math. Soc., 25 (1991), pp. 403-412.

[15] W. Menasco and M. Thistlethwaite, The classification of alternating links, Ann. Math., 138 (1993), pp. 113-171.

[16] K. Murasugi, Knot Theory \& Its Applications, Birkhäuser, Boston 1996.

[17] Y. Nakanishi, Unknotting numbers and knot diagrams with the minimum crossings, Math. Seminar Notes Kobe Univ. 11(2) (1983), pp. 257-258.

[18] D. Rolfsen, Knots and Links, 2nd ed., Math. Lect. Ser., 7. Publish or Perish, Inc., Houston, 1990.

[19] L. Rudolph, Quasipositivity as an obstruction to sliceness, Bull. Amer. Math. Soc. 29(1) (1993), pp. 51-59.

[20] A. Stoimenow, On the unknotting number of minimal diagrams, Mathematics of Computation, 72(244) (2003), pp. 2043-2057.

[21] A. Stoimenow, On unknotting numbers and knot trivadjacency, Mathematica Scandinavica 94 (2004), pp. 227-248.

[22] M.B. Thistlethwaite, Prime unoriented alternating links to 19 crossings, http://www. math.utk.edu/ morwen /png/link_stats.png. 
[23] I. Torisu, The Determination of the pairs of two bridge knots and links with Gordian distance one, Proc. AMS., 126(5), 1565-1571 (1998).

[24] P. Traczyk, A criterion for signed unknotting number, Contemporary Mathematics, 233 (1999), pp. 215-220.

Department of Mathematics, University of North Carolina at Charlotte, CharLOTTE, NC 28223, USA

E-mail address: ydiao@uncc.edu

Department of Mathematics, Western Kentucky University, Bowling Green, KY 42101, USA

E-mail address: claus.ernst@wku.edu

Laboratory of Ultrastructural Analysis, University of Lausanne, Lausanne, CH 1015, SwitzerLand

E-mail address: Andrzej.Stasiak@unil.ch 